University of Wollongong

Research Online

Australian Institute for Innovative Materials -

Papers

Australian Institute for Innovative Materials

$1-1-2015$

Terahertz probes of magnetic field induced spin reorientation in $\mathrm{YFeO} 3$ single crystal

Xian Lin

Shanghai University

Junjie Jiang

Shanghai University

Zuanming Jin

Shanghai University

Dongyang Wang

Tianjin University

Zhen Tian

Tianjin University

See next page for additional authors

Follow this and additional works at: https://ro.uow.edu.au/aiimpapers

Part of the Engineering Commons, and the Physical Sciences and Mathematics Commons

Research Online is the open access institutional repository for the University of Wollongong. For further information contact the UOW Library: research-pubs@uow.edu.au 


\title{
Terahertz probes of magnetic field induced spin reorientation in YFeO 3 single crystal
}

\begin{abstract}
Using the terahertz time-domain spectroscopy, we demonstrate the spin reorientation of a canted antiferromagnetic $\mathrm{YFeO}_{3}$ single crystal, by evaluating the temperature and magnetic field dependence of resonant frequency and amplitude for the quasi-ferromagnetic (FM) and quasi-antiferromagnetic modes (AFM), a deeper insight into the dynamics of spin reorientation in rare-earth orthoferrites is established. Due to the absence of $4 f$-electrons in $Y$ ion, the spin reorientation of Fe sublattices can only be induced by the applied magnetic field, rather than temperature. In agreement with the theoretical predication, the frequency of FM mode decreases with magnetic field. In addition, an obvious step of spin reorientation phase transition occurs with a relatively large applied magnetic field of $4 \mathrm{~T}$. By comparison with the family members of $\mathrm{RFeO}_{3}\left(\mathrm{R}=\mathrm{Y}^{3+}\right.$ or rare-earth ions), our results suggest that the chosen of $\mathrm{R}$ would tailor the dynamical rotation properties of Fe ions, leading to the designable spin switching in the orthoferrite antiferromagnetic systems.
\end{abstract}

\section{Keywords}

spin, reorientation, yfeo, 3 , single, probes, crystal, magnetic, terahertz, field, induced

\section{Disciplines}

Engineering | Physical Sciences and Mathematics

\section{Publication Details}

Lin, X., Jiang, J., Jin, Z., Wang, D., Tian, Z., Han, J., Cheng, Z. \& Ma, G. (2015). Terahertz probes of magnetic field induced spin reorientation in $\mathrm{YFeO}_{3}$ single crystal. Applied Physics Letters, 106 (9),

092403-1-092403-4.

\section{Authors}

Xian Lin, Junjie Jiang, Zuanming Jin, Dongyang Wang, Zhen Tian, Jiaguang Han, Zhenxiang Cheng, and Guohong Ma 


\title{
Terahertz probes of magnetic field induced spin reorientation in $\mathrm{YFeO}_{3}$ single crystal
}

\author{
Xian Lin, ${ }^{1}$ Junjie Jiang, ${ }^{1}$ Zuanming Jin, ${ }^{1,2}$ Dongyang Wang, ${ }^{3}$ Zhen Tian, ${ }^{3}$ Jiaguang Han, ${ }^{3}$ \\ Zhenxiang Cheng, ${ }^{1,4}$ and Guohong $\mathrm{Ma}^{1, \text { a })}$ \\ ${ }^{1}$ Department of Physics, Shanghai University, Shanghai 200444, People's Republic of China \\ ${ }^{2}$ Max Planck Institute for Polymer Research, Ackermannweg 10, 55128 Mainz, Germany \\ ${ }^{3}$ Center for Terahertz Waves and College of Precision Instrument and Optoelectronics Engineering, \\ Key Laboratory of Optoelectronics Information and Technology (Ministry of Education), \\ Tianjin University, Tianjin 300072, People's Republic of China \\ ${ }^{4}$ Institute for Superconducting and Electronic Materials, University of Wollongong, Wollongong, \\ New South Wales 2522, Australia
}

(Received 16 January 2015; accepted 19 February 2015; published online 3 March 2015)

\begin{abstract}
Using the terahertz time-domain spectroscopy, we demonstrate the spin reorientation of a canted antiferromagnetic $\mathrm{YFeO}_{3}$ single crystal, by evaluating the temperature and magnetic field dependence of resonant frequency and amplitude for the quasi-ferromagnetic (FM) and quasiantiferromagnetic modes (AFM), a deeper insight into the dynamics of spin reorientation in rare-earth orthoferrites is established. Due to the absence of $4 f$-electrons in $\mathrm{Y}$ ion, the spin reorientation of Fe sublattices can only be induced by the applied magnetic field, rather than temperature. In agreement with the theoretical predication, the frequency of FM mode decreases with magnetic field. In addition, an obvious step of spin reorientation phase transition occurs with a relatively large applied magnetic field of $4 \mathrm{~T}$. By comparison with the family members of $\mathrm{RFeO}_{3}\left(\mathrm{R}=\mathrm{Y}^{3+}\right.$ or rare-earth ions), our results suggest that the chosen of $\mathrm{R}$ would tailor the dynamical rotation properties of Fe ions, leading to the designable spin switching in the orthoferrite antiferromagnetic systems. (C) 2015 AIP Publishing LLC. [http://dx.doi.org/10.1063/1.4913998]
\end{abstract}

Motivated by the potential applications in spintronics and energy efficient information technology, external field induced spin reorientation transition (SRT) in rare-earth orthoferrites $\left(\mathrm{RFeO}_{3}, \mathrm{R}=\right.$ rare-earth ions or $\left.\mathrm{Y}\right)$ attracts lots of interests; ${ }^{1-5}$ for example, the designable spin switching and magnetic recording. A key topic is to understand how does the $\mathrm{R}$ ion work during the SRT process, not only required by the fundamental physics involving Dzyaloshinskii-Moriya (DM) as well as R and Fe ions interactions but also required by the appealing designable spin switching in the antiferromagnetic systems. So far, several experimental techniques such as neutron diffraction, ${ }^{6} \mathrm{AC}$ magnetic susceptibility measurements, ${ }^{7}$ nuclear magnetic resonance (NMR), ${ }^{8}$ ultrasound, ${ }^{9}$ and Mössbauer spectra ${ }^{10}$ were employed to study the SRT; yet, the probes of spin resonant excitation, coherent control, and dynamical switching of spins are heavily needed on sub-picosecond timescale. ${ }^{11-14}$

Recently, the magnetic component of terahertz $(\mathrm{THz})$ pulse has been employed to non-thermally excite and manipulate the spin precession through magnetic dipole coupling in $\mathrm{RFeO}_{3} \cdot{ }^{15,16}$ The temperature induced SRT in $\mathrm{ErFeO}_{3}$ and $\mathrm{NdFeO}_{3}$ has been investigated by employing THz-time domain spectroscopy (TDS) recently. ${ }^{17,18}$ Compared with the spin dynamics triggered by femtosecond laser pulse with inverse Faraday effect ${ }^{19,20}$ in which the electron transition and the heat caused by the high photon energy of the pulse is a hindrance to its future application, $\mathrm{THz}$ pulse is direct

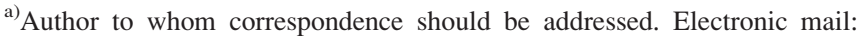
ghma@staff.shu.edu.cn
}

impulsive excitation of spin precession motion by the magnetic component of the terahertz pulse, is free from electronic excitations because of its much lower photon energy, in the meantime avoiding the thermal effect that could disturbs the spin system. Also, using terahertz time domain spectroscopy, more information can be extracted such as the amplitude, lifetime, and phase of spin systems via ultrafast time-domain analysis, which cannot be obtained from the static experiments.

As an antiferromagnetic $\mathrm{RFeO}_{3}$ crystal is illuminated by a THz pulse, the magnetic field component of the $\mathrm{THz}$ wave couples with the magnetic moment of the sample through a transient Zeeman torque $\mathbf{T}=\gamma \mathbf{M} \times \mathbf{H}_{\mathrm{THz}}$, where $\gamma$ denotes the gyromagnetic constant, $\mathbf{M}$ and $\mathbf{H}_{\mathrm{THz}}$ are the macroscopic magnetization and the impulsive magnetic field of $\mathrm{THz}$ wave, respectively. Tipped out of the easy direction by $\mathbf{T}$, the tilting magnetic moment $\mathbf{M}$ starts to process around the effective magnetic field, which persists within the spin relaxation time. Two optically active spin resonance modes, quasi-ferromagnetic mode (FM) and quasi-antiferromagnetic mode (AFM), can be selectively excited as the $\mathbf{H}_{\mathrm{THz}}$ aligns perpendicular and parallel to the $\mathbf{M}$, respectively. The induced precession of the macroscopic magnetization is expected to radiate free induction decay (FID) signal with the magnetic resonance frequency. The frequencies and amplitudes of both two modes are sensitive to the geometry between $\mathbf{H}_{\mathrm{THz}}$ and $\mathbf{M}$. The amplitude of the FID signal is proportional to $\mathbf{T}$, which has the largest value when $\mathbf{H}_{\mathrm{THz}}$ is perpendicular to $\mathbf{M}$. Importantly, one can therefore expect the magnitude change of $\mathbf{T}$ when SRT occurs (i.e., $\mathbf{M}$ deviates the orientation of perpendicular to $\mathbf{H}_{\mathrm{THz}}$ ). By recording 
the dependences of the spin resonance (such as amplitude and frequency) on temperature and magnetic field, detailed information about SRT can be obtained using THz-TDS.

As described by Dzyaloshinsky and Moriya, the low symmetry combined with spin-orbit coupling gives rise to anti-symmetric exchange interactions of $\mathrm{RFeO}_{3} \cdot{ }^{21,22}$ Below Néel temperature and absent of external magnetic field, the magnetic structure corresponds to the irreducible representation $\Gamma_{4}$ phase $\left(\mathrm{G}_{\mathrm{x}}, \mathrm{F}_{\mathrm{z}}\right)$ of iron orders with a weak ferromagnetic moment $\mathbf{F}$ along $c$-axis $(c \| z$, the $\mathbf{x}, \mathbf{y}, \mathbf{z}$ coordinate axes are set along $a, b$, and $c$ axes of the crystal, respectively). The magnetic moment along one of the symmetry axis of the crystal is known as a symmetric phase. ${ }^{23,24}$ The temperatureinduced spontaneous SRT in orthoferrites shows a sequence of transition $\Gamma_{4} \rightarrow \Gamma_{24} \rightarrow \Gamma_{2}\left(\mathrm{G}_{\mathrm{x}} \mathrm{F}_{\mathrm{z}} \rightarrow \mathrm{G}_{\mathrm{x}} \mathrm{F}_{\mathrm{z}} \mathrm{G}_{\mathrm{z}} \mathrm{F}_{\mathrm{x}} \rightarrow \mathrm{G}_{\mathrm{z}} \mathrm{F}_{\mathrm{x}}\right.$, where $\mathbf{F}$ and $\mathbf{G}$ are the ferro- and antiferro-magnetism vectors, respectively). The sample system is transferred into another symmetric configuration $\Gamma_{2}$ (F//a-axis). ${ }^{17,18,24}$ Two second-order phase transition points defines a temperature range of SRT. Temperature-induced spin switching is mainly caused by the competition between Fe ions ( $3 d$ electron subsystem) and paramagnetic $\mathrm{R}$ ions ( $4 f$ electron subsystem). On the other hand, the weak ferromagnetic moment $\mathbf{F}$ is expected to rotate from $c$-axis toward the direction of $\mathbf{H}_{\text {ext }}$ as an external magnetic field $\left(\mathbf{H}_{\text {ext }}\right)$ is applied along vector $\mathbf{G}$ $\left(\mathbf{H}_{\text {ext }} / / a\right.$-axis $)$, which yields a progressive increase of the magnetization along $a$-axis. ${ }^{25,26}$

As a family member of canted antiferromagnetic $\mathrm{RFeO}_{3}$, yttrium orthoferrites $\left(\mathrm{YFeO}_{3}\right)$ has no $f$ electron in $\mathrm{Y}^{3+}$ ions. Owing to the nonmagnetic characteristic of $\mathrm{Y}$ ions, $\mathrm{YFeO}_{3}$ is an excellent candidate for investigation of external magnetic field induced SRT of iron sublattice, avoiding the complex exchange interaction between rareearth ions and $\mathrm{Fe}$ ions under magnetic field, in particular. A single crystal of $\mathrm{YFeO}_{3}$ was grown by the optical floatingzone method. The sample ( $c$-cut, $1.33 \mathrm{~mm}$ thick) is polished on both sides for $\mathrm{THz}$ measurements. The all fiber-based THz-TDS integrated with the strong-magnetic field and a low-temperature system was used for TDS measurement. The emitter of the THz-TDS was excited by a $1560 \mathrm{~nm}$ laser pulse. The sample was mounted in the Oxford Instruments Spectromag He-bath cryostat (SHC) placed in a collimated terahertz beam. The $\mathrm{THz}$ pulse that transmitted through the sample was focused onto the receiver. The temperature ranges from 1.5 to $260 \mathrm{~K}$ and the magnetic field is up to the maximum value of $7 \mathrm{~T}$. The temperature- and magnetic fieldfluctuation of the SHC was well controlled within $0.01 \mathrm{~K}$ and $1 \mathrm{mT}$, respectively. The spectral range of the $\mathrm{THz}$ we used from 0.1 to $1.5 \mathrm{THz}$, centered at $0.6 \mathrm{THz}$ and the signal to noise ratio was 1000:1. The THz pulse scans were measured with a time range of $30 \mathrm{ps}$, corresponding to a spectral resolution of about $0.03 \mathrm{THz}$.

Typical THz-TDS in time-domain and its Fourier transform spectrum of $\mathrm{YFeO}_{3}$ have been represented in our previous papers and supplementary material. ${ }^{16,27,28}$ A zoomed-in section of the transmittance of $\mathrm{THz}$ waveform is shown in the inset of Fig. 1, a resonant dip at around $0.29 \mathrm{THz}$ corresponds to the FM mode at room temperature. Figure 1 presents the resonant frequency and amplitude of the FM spin-wave mode as a function of temperature. The resonant

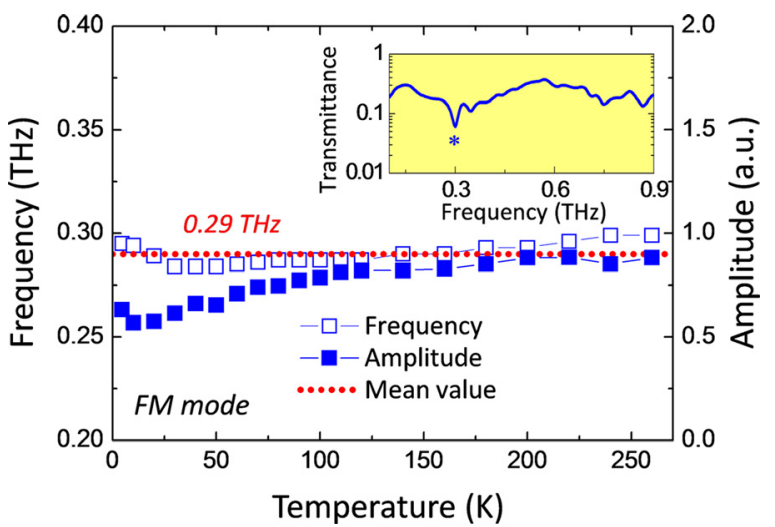

FIG. 1. The resonance frequency and the spectral amplitude of FM mode in the $c$-cut $\mathrm{YFeO}_{3}$ single crystal are shown as functions of temperature. The frequency is obtained from the dip in transmittance spectra (inset), which is calculated by the ratio of the Fourier transformed spectrum to a reference spectrum at room temperature.

frequency of FM mode shows mostly temperature independence with a mean value of $0.29 \mathrm{THz}$, as shown by the dashed line in Fig. 1. Additionally, the amplitude of FM mode decreases slightly as the sample is cooled. We do not observe any other resonances at higher frequency range, which means that AFM mode in $\mathrm{YFeO}_{3}$ has never been available for the c-cut sample in the whole temperature range. In contrast to the temperature-induced SRT in $\mathrm{NdFeO}_{3}$, the SRT of $\mathrm{Fe}^{3+}$ sublattice is ascribed to the exchange interaction between Nd- $4 f$ and $\mathrm{Fe}-3 d$ electrons, either theoretically or experimentally. ${ }^{18}$ Indeed, $\mathrm{SRT}$ in $\mathrm{YFeO}_{3}$ cannot be triggered by temperature, due to the absence of $4 \mathrm{f}$ electron in $\mathrm{Y}$ ion, which makes $\mathrm{YFeO}_{3}$ be different from other common rareearth orthoferrites, such as $\mathrm{ErFeO}_{3}, \mathrm{NdFeO}_{3}$, and $\mathrm{TmFeO}_{3}$, etc., in which the ferromagnetic vector $\mathbf{F}$ rotates by $90^{\circ}$ as the temperature is varied across characteristic SR temperatures, $\mathrm{YFeO}_{3}$ stays in the $\Gamma_{4}$ phase from $\mathrm{T}_{\mathrm{N}}$ to the temperature as low as a few Kelvin. Our THz-TDS data is consistent with previous measurements. ${ }^{29}$

In the following, we focus on the study of magnetic field induced SRT in $\mathrm{YFeO}_{3}$. The magnetic field of $\mathrm{THz}$ pulse and external magnetic field are set parallel to the $a$-axis of $\mathrm{YFeO}_{3}$ crystal $\left(\mathbf{H}_{\text {ext }} / / \mathbf{H}_{\mathrm{THz}} / / a\right.$-axis $)$. The time resolved signals are first converted into the frequency domain by Fourier transformation. The normalized loss function, $\alpha(\omega)$, including both reflective and absorptive losses, is given by Beer-Lambert law

$$
\alpha(\omega)=-\ln \left(\left|\frac{\mathrm{T}(\omega)}{\mathrm{T}_{\mathrm{ref}}(\omega)}\right|\right),
$$

where $\mathrm{T}(\omega)$ is the transmitted spectrum of the $\mathrm{THz}$ signal at different values of external magnetic field and $\mathrm{T}_{\text {ref }}(\omega)$ is the reference spectrum transmitted through the system without sample at zero magnetic field. According to Eq. (1), Figs. 2(a) and 2(b) show the amplitude mapping of the THz loss spectrum in two frequencies ranges from 0.48 to $0.6 \mathrm{THz}$ and 0.2 to $0.36 \mathrm{THz}$, respectively, at $260 \mathrm{~K}$. As shown in Fig. 2(b), the resonant absorption peak corresponding to the FM mode, which exhibits continuous softening from 0.29 to $0.21 \mathrm{THz}$, as the external magnetic field is increased from 0 to $7 \mathrm{~T}$. 


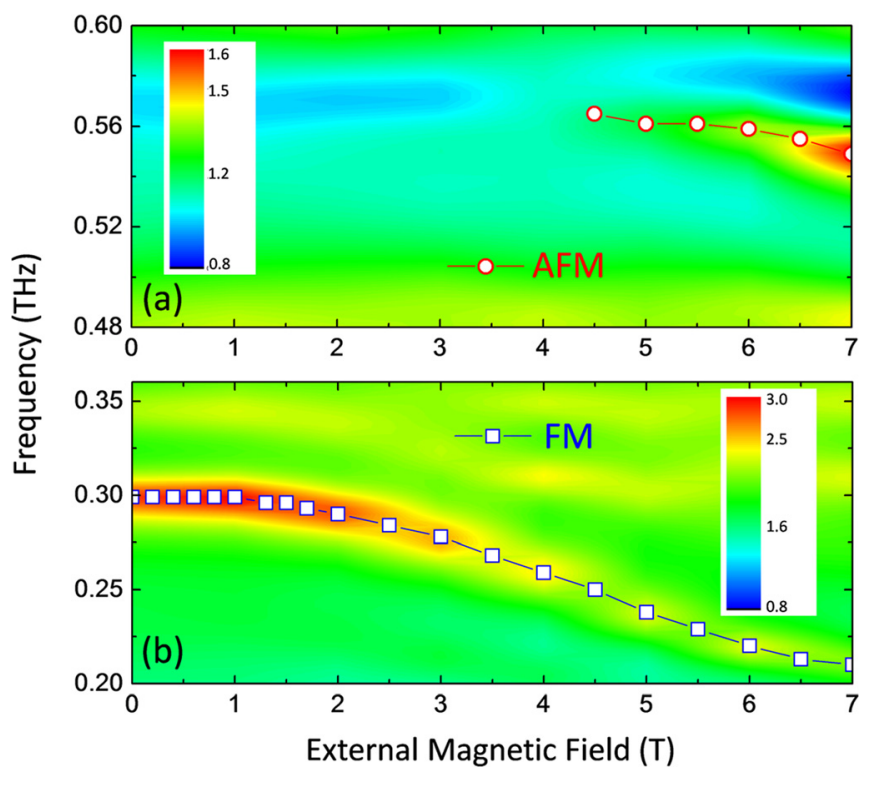

FIG. 2. The amplitude mapping of the loss function, as a function of the external magnetic field along $a$-axis, in the cases of two spectrum regions: AFM mode (a) and FM mode region (b) at temperature of $260 \mathrm{~K}$.

At lower applied magnetic field $(\mathrm{H}<2 \mathrm{~T})$, the frequency of FM mode keeps relative constant, which indicates that the phase of $\mathrm{YFeO}_{3}$ crystal changes slowly when the applied magnetic field is below $2 \mathrm{~T}$. We can hardly see any absorption peak in the high frequency range with lower magnetic field, as shown in Fig. 2(a). By increasing the magnitude of $\mathrm{H}$ above $2 \mathrm{~T}$, the frequency of FM-mode starts to decrease quickly with magnetic field, and the mode is still observable with the field up to $7 \mathrm{~T}$. The frequency softening of FMmode is indicative of the appearance of SRT induced by magnetic field. On the other hand, the FM-mode persists up to $7 \mathrm{~T}$ implies the SRT does not complete under the field of $7 \mathrm{~T}$. It is important to note that a higher resonant frequency at $0.55 \mathrm{THz}$ is clearly observed at the magnetic field of $4 \mathrm{~T}$, and this frequency mode decreases slightly with the magnetic field, as shown in Fig. 2(a). According to our experimental results, we can conclude that the $\mathrm{YFeO}_{3}$ is $\Gamma_{4}$ phase without magnetic field, and the crystal is transformed into $\Gamma_{24}$ phase when apply the magnetic field. Previous studies revealed that the SRT is completed under a critic magnetic field $\mathrm{H}_{\mathrm{cr}}=7.4 \mathrm{~T}^{30}$ As the highest magnetic field is $7 \mathrm{~T}$ in our system, the $\Gamma_{2}$ phase of $\mathrm{YFeO}_{3}$ cannot be reached in the present condition.

In a qualitative picture, as the magnetic-field of incident $\mathrm{THz}$ pulse is perpendicular to the vector $\mathbf{F}$ of $\mathrm{YFeO}_{3}$, the observed oscillation at a frequency of $0.29 \mathrm{THz}$ is due to the FM spin resonance mode. Under external magnetic field, as shown in the inset of Fig. 3(a), the magnetic moment rotates from the $c$-axis to $a$-axis in $a c$-plane, corresponding to the SRT from $\Gamma_{4}$ to $\Gamma_{2}$ via $\Gamma_{24}$. Our experimental observation of the oscillation at $0.55 \mathrm{THz}$, therefore, can be explained by the excitation of AFM spin resonance mode, as the vector $\mathbf{F}$ has the component along the magnetic-field of $\mathrm{THz}$ pulse.

This reorientation behavior can also be observed in Fig. 3(a), which shows the magnetic-field dependent spectral amplitudes of FM and AFM modes. The spectral amplitude here is extracted by the full width at half maximum
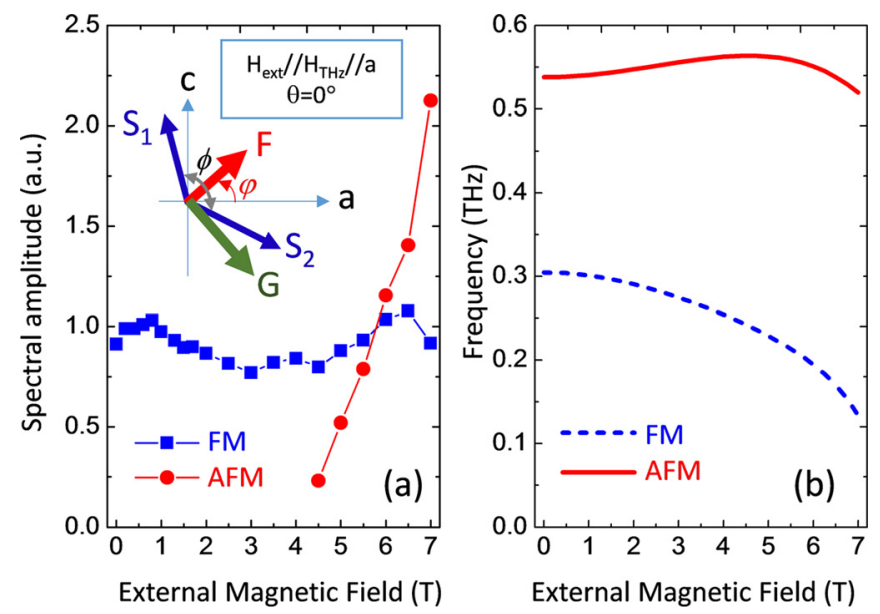

FIG. 3. (a) Spectral amplitudes of FM mode (blue squares) and AFM mode (red dots) are shown as functions of applied magnetic field. Inset shows the sketch of the simplified magnetic configuration in $\mathrm{YFeO}_{3}$ upon the spin reorientation process. $\mathbf{S}_{1}$ and $\mathbf{S}_{2}$ are two pairs of spins for $\mathrm{Fe}^{3+}$ sublattices. (b) Results of the calculation of the frequencies of the FM and AFM modes as functions of external magnetic field, where the $\mathbf{H}_{\text {ext }}$ applied along the $a$-axis at $293 \mathrm{~K}$. The parameters used in the calculation are $\mathrm{H}_{\mathrm{E}}=6.4 \times 10^{6} \mathrm{Oe}$, $\mathrm{H}_{\mathrm{D}}=1.4 \times 10^{5} \mathrm{Oe}, \eta=0.7, \mathrm{H}_{\mathrm{ac}}=1.8 \times 10^{3} \mathrm{Oe}, \mathrm{H}_{\mathrm{A} 2}=3.3 \times 10^{2} \mathrm{Oe}$, and $\mathrm{H}_{\mathrm{E}} \mathrm{H}_{\mathrm{ac}}=11600 \mathrm{kOe}^{2}$, respectively.

(FWHM) of the corresponding absorption peaks. The spectral amplitude of AFM mode (circles) increases significantly as the magnetic field is larger than $4 \mathrm{~T}$. It is consistent with the emergence of detectable AFM mode (Fig. 2(a)) and suggests an acceleration of SRT process in $\mathrm{YFeO}_{3}$ with a relatively large applied magnetic field. As expected from the magnetic field induced SRT (inset of Fig. 3(a)), the transient torque $\mathbf{T}$ is seen to decrease when the vector $\mathbf{F}$ rotates from $c$-axis to $a$-axis. Thus, the spectral amplitude of FM mode is expected to be decreased with an increasing magnetic field along $a$-axis. However, one notices that the spectral amplitude of FM mode does not change a lot within our magnetic field range. In other words, our experimental results suggest that the component vector of $\mathbf{F}$ along $c$-axis is kept constant. The explanation is, apart from the angle $\varphi$ between the vector $\mathbf{F}$ and $a$-axis becomes smaller during the SRT, the evolution of angle $\phi$ between vectors $\mathbf{S}_{1}$ and $\mathbf{S}_{2}$ cannot be ruled out, for the external magnetic field-induced SRT. Our measurements reveal a balance between these two contributions in $\mathrm{YFeO}_{3}$ with an increasing magnetic field. In contrast, $\phi$ is in principle considered as constant within the SRT induced by temperature. Limited by our experimental condition, the maximum value of the magnetic field is up to $7 \mathrm{~T}$ along $a$-axis, the spins are mainly switched from $\Gamma_{4}$ to $\Gamma_{24}$. Even larger magnetic field of around 7.4 $\mathrm{T}$ was used to completely perform the SRT from $\Gamma_{4}$ to $\Gamma_{2}{ }^{30}$

To better understand the SRT in $\mathrm{YFeO}_{3}$ under external magnetic field in a quantitative picture, a simplified thermodynamic potential $\Phi$, based on two-sublattice approximation is then expected as 31

$$
\Phi=\mathrm{A}\left(\mathbf{S}_{1} \mathbf{S}_{2}\right)+\frac{1}{2} a\left(S_{1 z}^{2} S_{2 z}^{2}\right)+b S_{1 z} S_{2 z}-\mathbf{S}_{1} \mathbf{H}+\mathbf{S}_{2} \mathbf{H}
$$

where $\mathrm{A}$ is the exchange interaction parameter, $\mathrm{a}$ and $\mathrm{b}$ are the anisotropy parameters. In the case of a magnetic field 
along the $a$-axis (i.e., $\theta=0$ ), the equilibrium values of the angle $\varphi$ are given by ${ }^{32,33}$

$$
\begin{aligned}
\left(\frac{\partial \Phi}{\partial \varphi}\right)_{\mathrm{M}=M_{0}, \theta=0}= & \cos \varphi\left(H_{E} H_{a c} \sin \varphi+H_{E} H_{A 2} \sin ^{3} \varphi\right. \\
& \left.-\eta H^{2} \sin \varphi-H H_{D}\right)=0,
\end{aligned}
$$

where $\mathrm{H}_{\mathrm{E}}$ is the effective symmetric exchange field, $\mathrm{H}_{\mathrm{ac}}$ is the effective bilinear anisotropy fields in ac plane, $\mathrm{H}_{\mathrm{A} 2}$ is the effective biquadratic anisotropy fields in $a c$ plane, $\mathrm{H}_{\mathrm{D}}$ is the antisymmetric exchange field, and $\eta=\left(\chi_{\perp}-\chi_{/ /}\right) / \chi_{\perp}, \chi_{\perp}$ and $\chi_{/ /}$are the longitudinal and transverse antiferromagnetic susceptibilities, respectively. When $\mathrm{H} \leq \mathrm{H}_{\mathrm{cr}}$, the sample system stays in the canted phase, the angle $\varphi$ of the canted phase is governed by $\mathrm{H}^{32,33}$

$$
H_{E} H_{A 2} \sin ^{3} \varphi+\left(H_{E} H_{a c}-\eta H^{2}\right) \sin \varphi-H H_{D}=0 .
$$

The dynamical spin response can be solved by the Landau-Lifshitz-Gilbert (LLG) equation, detailed theory calculation can be found in supplementary material. ${ }^{28}$ Figure 3(b) shows the results of calculated frequencies of FM (dashed line) and AFM mode (solid line) in the canted phase $\left(\mathrm{H} \leq \mathrm{H}_{\mathrm{cr}}\right)$, as a function of external magnetic field. The parameters we used here are $\mathrm{H}_{\mathrm{E}}=6.4 \times 10^{6} \mathrm{Oe}, \mathrm{H}_{\mathrm{D}}=1.4 \times$ $10^{5} \mathrm{Oe}, \eta=0.7, \mathrm{H}_{\mathrm{ac}}=1.8 \times 10^{3} \mathrm{Oe}, \mathrm{H}_{\mathrm{A} 2}=3.3 \times 10^{2} \mathrm{Oe}$, and $\mathrm{H}_{\mathrm{E}} \mathrm{H}_{\mathrm{ac}}=11600 \mathrm{kOe}^{2}{ }^{33}$ When we increase the external magnetic field, the frequency of FM mode decreases, which is well consistent with the data of Fig. 2. The frequency of AFM increases first, and then decreases within the whole magnetic field range. In this work, a resonant frequency at about $0.54 \mathrm{THz}$ is observed with high magnetic fields above $4 \mathrm{~T}$. Given that the AFM mode $(0.527 \mathrm{THz})$ has been reported with zero external magnetic field at room temperature, ${ }^{15}$ we therefore expect the frequency of AFM mode has a slight increased tendency as the magnetic field is increased to $4 \mathrm{~T}$, which coincides with our calculation results. Our experimental results emphasize that the observable SRT occurs as the external magnetic field is larger than $4 \mathrm{~T}$.

The similar experiments have been also performed on a $\mathrm{NdFeO}_{3}$ single crystal. However, one can observe different spin switching. For example, cooling of $\mathrm{NdFeO}_{3}$ from 170 to $100 \mathrm{~K}$ leads to a continuous rotation of $\mathbf{F}$ from $c$ - to $a$-axis and an irreversible switching between $\Gamma_{4}$ and $\Gamma_{2}$ is attributed to the anisotropy of $\mathrm{Nd}^{3+}$. Our findings here indicate that $\mathrm{Y}$ or rare-earth ions play an important role in the SRT of orthoferrite antiferromagnetic single crystal.

In summary, the THz-TDS has been used for investigation of the dynamical SRT in $\mathrm{YFeO}_{3}$ single crystal. Owing to be absence of $4 f$-electrons in $\mathrm{Y}$ ion, the spin reorientation of Fe sublattices can only be induced by the applied magnetic field, rather than temperature. Our data are in agreement with a simplified thermodynamic model with two-sublattice approximation. Furthermore, compared with the magnetic field-induced SRT of $\mathrm{NdFeO}_{3}$, we clearly demonstrate the difference of spin switching of Fe ions. Given that the anisotropy can be significantly modified by rare-earth ions, we foresee lots of opportunities to design spin switching in the orthoferrite systems.
The research was supported by National Natural Science Foundation of China (Nos. 11174195, 50932003, 11274221), Ph.D. Programs Foundation of Ministry of Education of China (No. 20123108110003), and the Research Innovation Fund of the Shanghai Education Committee (No. 14ZZ101).

${ }^{1}$ G. Cinader, Phys. Rev. 155, 453 (1967).

${ }^{2}$ M. D. Lumsden, B. C. Sales, D. Mandrus, S. E. Nagler, and J. R. Thompson, Phys. Rev. Lett. 86, 159 (2001).

${ }^{3}$ M. R. Moldover, G. Sjolander, and W. Weyhmann, Phys. Rev. Lett. 26, 1257 (1971).

${ }^{4}$ J. Scola, Y. Dumont, N. Keller, M. Vallée, J. G. Caputo, I. Sheikin, P. Lejay, and A. Pautrat, Phys. Rev. B 84, 104429 (2011).

${ }^{5}$ Y. Cao, S. Cao, W. Ren, Z. Feng, S. Yuan, B. Kang, B. Lu, and J. Zhang, Appl. Phys. Lett. 104, 232405 (2014).

${ }^{6}$ W. C. Koehler, E. O. Wollan, and M. K. Wilkinson, Phys. Rev. 118, 58 (1960).

${ }^{7}$ H. Shen, Z. Cheng, F. Hong, J. Xu, S. Yuan, S. Cao, and X. Wang, Appl. Phys. Lett. 103, 192404 (2013).

${ }^{8}$ S. N. Barilo, Sov. Phys. Solid State 33, 354 (1991).

${ }^{9}$ L. T. Tsymbal, Y. B. Bazaliy, V. N. Derkachenko, V. I. Kamenev, G. N. Kakazei, F. J. Palomares, and P. E. Wigen, J. Appl. Phys. 101, 123919 (2007).

${ }^{10}$ S. R. Brown and I. Hall, J. Phys.: Condens. Matter 5, 4207 (1993).

${ }^{11}$ A. V. Kimel, A. Kirilyuk, P. A. Usachev, R. V. Pisarev, A. M. Balbashov, and Th. Rasing, Nature 435, 655 (2005).

${ }^{12}$ A. Kirilyuk, A. V. Kimel, and Th. Rasing, Rev. Mod. Phys. 82, 2731 (2010).

${ }^{13}$ A. V. Kimel, B. A. Ivanov, R. V. Pisarev, P. A. Usachev, A. Kirilyuk, and T. Rasing, Nat. Phys. 5, 727 (2009).

${ }^{14}$ T. Kampfrath, A. Sell, G. Klatt, A. Pashkin, S. Mahrlein, T. Dekorsy, M. Wolf, M. Fiebig, A. Leitenstorfer, and R. Huber, Nat. Photonics 5, 31 (2011).

${ }^{15}$ K. Yamaguchi, M. Nakajima, and T. Suemoto, Phys. Rev. Lett. 105, 237201 (2010).

${ }^{16}$ Z. Jin, Z. Mics, G. Ma, Z. Cheng, M. Bonn, and D. Turchinovich, Phys. Rev. B 87, 094422 (2013).

${ }^{17}$ K. Yamaguchi, T. Kurihara, Y. Minami, M. Nakajima, and T. Suemoto, Phys. Rev. Lett. 110, 137204 (2013).

${ }^{18}$ J. J. Jiang, Z. M. Jin, G. B. Song, X. Lin, G. H. Ma, and S. X. Cao, Appl. Phys. Lett. 103, 062403 (2013).

${ }^{19}$ A. V. Kimel, A. Kirilyuk, and T. Rasing, Laser Photon. Rev. 1, 275 (2007).

${ }^{20}$ Z. M. Jin, H. Ma, L. H. Wang, G. H. Ma, F. Y. Guo, and J. Z. Chen, Appl. Phys. Lett. 96, 201108 (2010).

${ }^{21}$ I. Dzyaloshinsky, J. Phys. Chem. Solids 4, 241 (1958).

${ }^{22}$ T. Moriya, Phys. Rev. 120, 91 (1960).

${ }^{23}$ H. Luttgemeir, H. G. Bohn, and M. Brajczewska, J. Magn. Magn. Mater. 21, 289 (1980).

${ }^{24}$ Y. B. Bazaliy, L. T. Tsymbal, G. N. Kakazei, A. I. Izotov, and P. E. Wigen, Phys. Rev. B 69, 104429 (2004).

${ }^{25}$ W. Slawinski, R. Przenioslo, I. Sosnowska, and E. Suard, J. Phys.: Condens. Matter 17, 4605 (2005).

${ }^{26}$ G. W. Durbin, C. E. Johnson, and M. F. Thomas, J. Phys. C: Solid State Phys. 8, 3051 (1975).

${ }^{27}$ R. Z. Zhou, Z. M. Jin, G. F. Li, G. H. Ma, Z. X. Cheng, and X. L. Wang, Appl. Phys. Lett. 100, 061102 (2012).

${ }^{28}$ See supplementary material at http://dx.doi.org/10.1063/1.4913998 for typical time domain spectroscopy, its Fourier transform spectroscopy and calculation of the resonant frequencies.

${ }^{29}$ A. A. Volkov, Yu. G. Goncharov, G. V. Kozlov, K. N. Kocharyan, S. P. Lebedev, A. S. Prokhorov, and A. M. Prokhorov, Pis'ma Zh. Eksp. Teor. Fiz. 39, 140 (1984) [JETP Lett. 39, 166 (1984)].

${ }^{30}$ J. Scola, W. Noun, E. Popova, A. Fouchet, Y. Dumont, and N. Keller, Phys. Rev. B 81, 174409 (2010).

${ }^{31}$ Y. A. Izyumov and V. N. Syromyatnikov, Phase Transition and Crystal Symmetry (Springer, Berlin, 1990).

${ }^{32}$ I. S. Jacobs, H. F. Burne, and L. M. Levinson, J. Appl. Phys. 42, 1631 (1971).

${ }^{33}$ A. M. Balbashov, A. G. Berezin, Yu. M. Gufan, G. S. Kolyadko, P. Yu. Marchukov, and E. G. Rudashevsky, Sov. Phys. JETP 66, 174 (1987). 